Pacific Journal of Mathematics

THE GROUP-VALUED LEBESGUE DECOMPOSITION 


\title{
THE GROUP-VALUED LEBESGUE DECOMPOSITION
}

\author{
TIM TRAYNOR
}

\begin{abstract}
An s-bounded additive map on a topological Boolean algebra to a topological group can be decomposed into a continuous and a singular part. This can be done in a canonical way as a limit thoerem in spaces of oprators. As a consequence, if $\mathscr{A}$ is a Boolean algebra of continuous projections on a (complete) topological group $X$ and $\mathscr{G}$ is a "FréchetNikodým" topology on $\mathscr{A}$, then every $x$ in $X$, viewed as an additive map $A \rightarrow A x$ on $\mathscr{A}$, can be decomposed uniquely as the sum of a $\mathscr{G}$-continuous and a $\mathscr{G}$-singular part. If $\mathscr{A}$ is equicontinuous, the operators which decompose $x$ are continuous. The result applies to the space of all $s$-bounded additive functions on an algebra of sets to a complete separated topological group.
\end{abstract}

In 1963, R. B. Darst [4] published a proof of a Lebesgue-type decomposition theorem for complete normed abelian groups. The result extended C. E. Rickart's theorem [12] on decomposition of Banach-space valued set functions. It also turned out to contain subsequent results of J. J. Uhl [14] and of J. K. Brooks [2]. This was shown in detail by T. P. Dence [6]. About the same time, Darst [5] indicated how his work carried over to lattices of projection operators, and hence to modular functions defined on a lattice of sets. The purpose of this note is to show how the results and methods of [13] extend and shed light on this and related work. In particular, we find that the topology on the group need not be metrizable and that the same goes for the notion of convergence on the algebra (it is metrizable in Darst's work). (In passing, we see that a hypothesis of Darst's result can be removed so that it applies to join semilattices.) The results may be formulated as limit theorems in spaces of operators, so as to apply to all "decomposable" elements simultaneously.

Let $X$ be a commutative separated topological group and . $\mathscr{A}$ a Boolean algebra (with operations noted by the corresponding set theoretic symbols). Put a topology $\mathscr{G}$ on $\mathscr{A}$ such that $\mathrm{A} B=\mathrm{A} \cap B$ is continuous in $A$ uniformly for $B$ in $\mathscr{A}$ and that $A \triangle B$ is jointly continuous in $A$ and $B$ (an $F N$-topology in the sense of Drewnowski [8]). A map $m$ on $\mathscr{C}$ to $X$ is called $\mathscr{G}$-continuous if it is continuous in the topology $\mathscr{C}$ and is called $\mathscr{G}$-singular if for each neighborhood $U$ of 0 in $X$ and each $\mathscr{G}$-neighborhood $G$ of 0 in $\mathscr{A}$, there exists $A$ in $G$ with $m\left(\left(A^{c}\right)\right)=\left\{m\left(A^{c} E\right): E \in \mathscr{A}\right\} \subset U . \quad m$ is called sideways continuous [1] $(=s$-bounded, $=$ exhaustive $)$, provided $m A_{i} \rightarrow 0$, whenever 
$\left(A_{i}\right)$ is a disjoint sequence in $\mathscr{\%}$ An additive sideways continuous map is strongly additive [7], and we shall use this terminology.

In the special case that $\mathscr{A}$ is a Boolean algebra of projections (that is, idempotent endomorphisms) on $X$ [3], to each $x$ in $X$ corresponds an additive map on $\mathscr{C}$ by evaluation $m: A \mapsto A x$. In this situation, $x$ is called $\mathscr{C}$-continuous or $\mathscr{S}$-singular or sideways continuous provided $m$ is. [Meet here is composition; the unit is $I$ (the identity map); $A^{c}=I-A$ (pointwise difference); $A \cup B=A+B-A B$.]

Here is the generalization of Darst's theorem:

THeOREm. Let $\mathscr{A}$ be a Boolean algebra of continuous projection operators on the commutative separated topological group $X$ and let $\mathscr{C}$ be an $F N$-topology on $\%$ Then any sideways continuous $x$ in $X$ with $\mathscr{A} x$ in a complete subset of $X$ may be uniquely written as the sum of a C्S-continuous and a C्S-singular member of $X$ (both sideways continuous).

(The proof will be given presently.)

To make it clear how Darst's result follows from this, for each

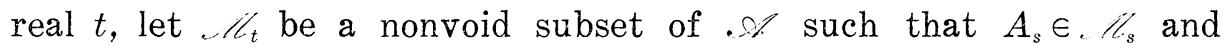
$A_{t} \in \mathscr{L}_{t}$ imply $A_{s} \cup A_{t} \in \mathscr{C l}_{s+t}$ (condition (5) of Darst [5] or (2) of [4]). We may define an $F N$-topology by taking for basic neighborhoods of 0 the families $\mathscr{Y}_{s}=\left\{A \in \mathscr{K}: A\right.$ is contained in a member of $\left.\mathscr{C}_{s}\right\}$. (Neighborhoods of the other members of . $/$ are obtained by translation under symmetric difference.) Since this topology has a countable local base, it is metrizable, so the present hypothesis-- not requiring metrizability - is the more general.

An $F N$-topology can be generated by a family $Q$ of submeasures (monotone, subadditive, real-valued functions $q$ with $q(0)=0$ ), in that the families $\{A \in \mathscr{C}: q(A)<\varepsilon\}(q \in Q, \varepsilon>0)$ form basic neighborhoods of 0 : see, for example, [8, 12.7] or [13]. The use of the families U/s amounts to using one submeasure $\left(q(A)=\inf \left\{s: A \in \mathscr{C}_{s}\right\}\right)$ to define the topology.

The condition (4) of Darst [5] (or (1) of [4]) is not used. As a matter of fact, Darst himself is not really using it-or rather it is implicit in the other conditions-in that his condition on the norm $(A \leqq B \Rightarrow\|A x\| \leqq\|B x\|)$ allows one to replace. $/ / l_{s}$ by $\mathscr{O}_{s}$ without affecting $\sup _{A \in \ldots}\|A g\|$. Thus the lattice $\mathscr{C l}$ of Darst [5] is not needed; the hypothesis is only on the join semilattice $U_{s}$. $/ / s$. (See however remark (4) below.)

Proof of theorem. By Theorem 1.2' of [13] (or rather by the Boolean algebra analogue thereof) $m: A \mapsto A x$ may be decomposed uniquely as a sum $C m+S m$ where $C m$ is $C$-continuous and $S m$ is 
$\mathscr{G}$-singular; morover, $\mathrm{Cm}$ and $\mathrm{Sm}$ are given by

$$
(C m) A=\lim _{i} m A E_{i}^{e} \text { and }(S m) A=\lim _{i} m A E_{i},
$$

where $\left(E_{i}\right)$ is a certain (triple iterated limit) net in $\mathscr{A}$. These limits are uniform in $A$. Since each member of $\mathscr{A}$ is assumed continuous, we see that $C m$ and $S m$ are induced by elements of $X$. Indeed, $C m A=\lim _{i} m A E_{i}^{c}=\lim _{i} A E_{i}^{c} x=A\left(\lim _{i} E_{i}^{c} x\right)$; and $S m A\left(\lim _{i} E_{i} x\right)$. Thus $x=\lim _{i} E_{i}^{c} x+\lim _{i} E_{i} x$ is the required decomposition. Notice that these components belong to closure $(. / 2 x)$, which is why no more completeness is required.

We see from the proof that we can say a little more:

(1) If $\mathscr{A} X$ is precomplete then the nets $\left(E_{i}^{c}\right)$ and $\left(E_{i}\right)$ converge pointwise on the subgroup $X_{0}$ of sideways continuous elements to disjoint projections $C$ and $S$ whose ranges form a direct decomposition of $X_{0}$ into its $\mathscr{G}$-continuous and $\mathscr{G}$-singular elements.

(2) If $\mathscr{A}$ is equicontinuous and $X$ is complete, then the subgroup $X_{0}$ of sideways continuous elements of $X$ is closed, hence also complete. Replacing $X$ by $X_{0}$ we can assume all members of $X$ are sideways continuous. Now, we could call the topology of pointwise convergence on the group $B(X)$ of all continuous endomorphisms of $X$ the "strong operator topology", in analogy with Banach space

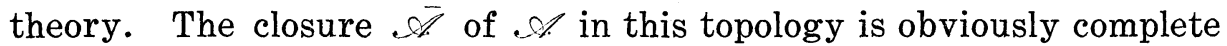
because of the equicontinuity. In particular, the operators $C$ and $S$ which decompose $X$ are continuous. Notice also that $\mathscr{A}$ is also complete as a Boolean algebra and that each additive map $A \mapsto A x$ is automatically extended to $\overline{\mathscr{h}}$.

Darst's work was inspired by spaces of measures, and perhaps a better feel for the significance of these remarks would be obtained by looking at such spaces. We are thinking along the lines referred to in Darst [4] and [5] and more explicitly set out in [3] and in Huff [11]. (The paper by Huff is partly responsible for our taking the operator point of view here.) We work, however, with group-valued set functions rather than scalar or Banach-space valued ones.

Let $Y$ be a complete commutative separated topological group, and let $A$ be a Boolean algebra of sets. Let $X$ be the group of strongly additive (=s-bounded additive) functions on $\mathscr{C}$ to $Y$ under the topology of uniform convergence on $\mathscr{A}$. Then $X$ is complete. Each member $A$ of $\mathscr{A}$ induces a projection operator $A^{*}$ on $X$ by restriction: $A^{\cdot} x(E)=x_{A} E=x(A E)$. The Boolean algebra $\mathscr{A}^{\cdot}$ of all such projections is isomorphic to $\mathscr{A}$ modulo those members $N$ of $\mathscr{C}$ which are null for all $x$ in $X$ (in the sense that $x(N E)=0$, for all $E$ in $\mathscr{C}$ and all $x$ in $X$ ). The usual indirect argument shows 
that each $x$ remains sideways continuous with respect to the algebra .. . Moreover, $X$ has a base for its neighborhoods of 0 consisting of closed symmetric sets invariant under $\mathscr{\varkappa}^{\cdot}$, in particular $\mathscr{L}^{\cdot}$ is equicontinuous. Thus the preceding results apply to this situation:

Corollary. Let $X$ be the group of strongly additive functions on an algebra. $\mathscr{\text { of }}$ sets to a commutative complete separated topological group, under the topology of uniform convergence on . $\%$ Then for each FN-topology $\mathscr{S}$ on.$S_{\text {th }}$ there exist continuous projections $C$ and $S$ providing a direct decomposition of $X$ into its subgroups of $\mathscr{C}$-continuous and $\mathscr{C}$-singular members. The operators $C$ and $S$ belong to the strong operator closure of the algebra of projections induced on $X$ by.$\%$.

(3) In the setting of the corollary, each element $x$ of $X$ induces an additive $x^{*}$ of $\mathscr{C}^{\cdot}$ by $x^{\bullet}\left(A^{*}\right)=x(A)$. The members of $\mathscr{A}^{\cdot}$ are of course dense in the complete Boolean algebra.$\overline{7}$. obtained by closing the former in the "strong operator topology". Since the maps $x^{*}$ are uniformly continuous in this topology, they may be extended by continuity to all of $\overline{\%}$. We thus have a more specific version of the type of extension sketched by Drewnowski at the end of [10].

(4) More needs to be said about the extent to which the results apply to lattices. If one starts with a modular set function on a lattice (containing, for simplicity, the whole space), it extends to an additive set function on the generated algebra. The result of Darst [5] and our extension herein apply only if the sideways continuity is assumed on the whole algebra. This condition does not carry over to the algebra from the lattice. The question therefore, is "What conditions on the function on the lattice are sufficient to obtain a similar decomposition?". In [13] (upon which this note is based) we actually used sideways continuity in the form of a monotone convergence condition: for each monotone sequence $\left(A_{i}\right)$ in $\mathscr{S}, m\left(A_{i} E\right)$ is Cauchy, uniformly for $E$ in $\%$. While this paper was being written, Isidore Fleischer informed me that the construction of [13] actually can be carried out under such a condition for modular functions on any distributive lattice.

\section{REFERENCES}

1. V. N. Aleksyuk and P. D. Beznosikov, Continuation of a vector measure on a Boolean algebra, Iz. Vysš. Učebn. Zaved. Mat. (Soviet Math. IzVUZ), 18 (1974), 3-7.

2. J. K. Brooks, Decomposition theorems for vector measures, Proc. Amer. Math. Soc., 21 (1969), 27-29.

3. R. B. Darst, $A$ decomposition for complete normed abelian groups with applications to spaces of additive set functions, Trans. Amer. Math. Soc., 103 (1962), 549-559. 
4. R. B. Darst, The Lebesgue decomposition, Duke Math. J., 30 (1963), 553-556.

5. - The Lebesgue decomposition for lattices of projection operators, Advances in Math., 17 (1975), 30-33.

6. T. P. Dence, A Lebesgue decomposition for vector-valued set functions, Pacific J. Math., 57 (1975), 91-98.

7. J. Diestel and J. J. Uhl, Vector measures, Amer. Math. Soc. Math. Surveys, 15 (1977).

8. L. Drewnowski, Topological rings of sets, continuous set functions, integration, I, II, III, Bull. Acad. Polon. Sci. Ser. Sci. Math. Astron. Phys., 20 (1972), 269-276, 277-286, 439-445.

9. - Decomposition of set functions, Studia Math., 48 (1973), 23-48.

10. - On complete submeasures, Comment. Math., 43 (1975), 177-186.

11. R. E. Huff, The Yosida-Hewitt decomposition as an ergodic theorem, Vector and Operator Valued Measures and Applications, Academic Press, (1973), 128-134.

12. C. E. Rickart, Decomposition of additive set functions, Duke Math. J., 10 (1943), 653-665.

13. T. Traynor, The Lebesgue decomposition for group-valued set functions, Trans. Amer. Math. Soc., 220 (1976), 307-319.

14. J. J. Uhl, Extension and decompositions of vector measures, J. Lond. Math. Soc., 3 (1971), 672-676.

Received July 8, 1977.

UNIVERSITY OF WINDSOR

WINDSOR, ONTARIO N9B 3P4 



\section{PACIFIC JOURNAL OF MATHEMATICS}

\section{EDITORS}

RICHARD ARENS (Managing Editor)

University of California

Los Angeles, California 90024

C. W. CURTIS

University of Oregon

Eugene, OR 97403

C. C. MOORE

University of California

Berkeley, CA 94720
J. DugundjI

Department of Mathematics University of Southern Californı Los Angeles, California 90007

R. Finn and J. Milgram Stanford University Stanford, California 94305

ASSOCIATE EDITORS

E. F. BECKENBACH

B. H. NeUmanN

F. WOLF

K. YoSHIDA

\section{SUPPORTING INSTITUTIONS}

UNIVERSITY OF BRITISH COLUMBIA CALIFORNIA INSTITUTE OF TECHNOLOGY

UNIVERSITY OF CALIFORNIA

MONTANA STATE UNIVERSITY

UNIVERSITY OF NEVADA, RENO

NEW MEXICO STATE UNIVERSITY

OREGON STATE UNIVERSITY

UNIVERSITY OF OREGON
UNIVERSITY OF SOUTHERN CALIFORNIA

STANFORD UNIVERSITY

UNIVERSITY OF HAWAII

UNIVERSITY OF TOKYO

UNIVERSITY OF UTAH

WASHINGTON STATE UNIVERSITY

UNIVERSITY OF WASHINGTON 


\section{Pacific Journal of Mathematics}

\section{Vol. 80, No. $1 \quad$ September, 1979}

Jeroen Bruijning and Jun-iti Nagata, A characterization of covering dimension by

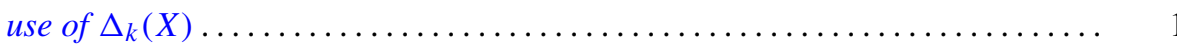

John J. Buoni and Albert Jonathan Klein, On the generalized Calkin algebra ...... 9

Thomas Ashland Chapman, Homotopy conditions which detect simple homotopy

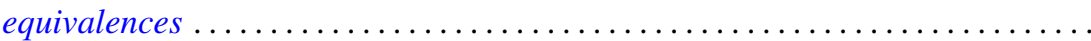

John Albert Chatfield, Solution for an integral equation with continuous interval

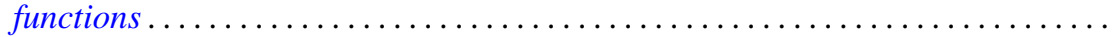

Ajit Kaur Chilana and Ajay Kumar, Spectral synthesis in Segal algebras on

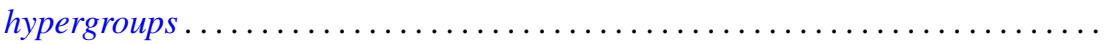

Lung O. Chung, Jiang Luh and Anthony N. Richoux, Derivations and

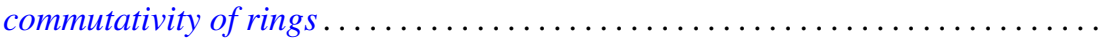

Michael George Cowling and Paul Rodway, Restrictions of certain function spaces to closed subgroups of locally compact groups .....................

David Dixon, The fundamental divisor of normal double points of surfaces........

Hans Georg Feichtinger, Colin C. Graham and Eric Howard Lakien,

Nonfactorization in commutative, weakly selfadjoint Banach algebras . . . . . . .

Michael Freedman, Cancelling 1-handles and some topological imbeddings ....... .

Frank E., III Gerth, The Iwasawa invariant $\mu$ for quadratic fields . . . . . . . . . . . . . .

Maurice Gilmore, Three-dimensional open books constructed from the identity

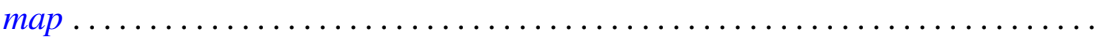

Stanley P. Gudder, A Radon-Nikodým theorem for $*$-algebras .

Peter Wamer Harley, III and George Frank McNulty, When is a point Borel? .

Charles Henry Heiberg, Fourier series with bounded convolution powers . .

Rebecca A. Herb, Characters of averaged discrete series on semisimple real Lie

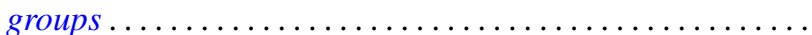

Hideo Imai, On singular indices of rotation free densities . .

Sushil Jajodia, On 2-dimensional CW-complexes with a single 2-cell . . .

Herbert Meyer Kamowitz, Compact operators of the form $u C_{\varphi}$

Matthew Liu and Billy E. Rhoades, Some properties of the Chebyshev method...

213

George Edgar Parker, Semigroups of continuous transformations and generating

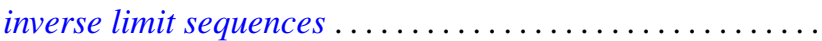

Samuel Murray Rankin, III, Oscillation results for a nonhomogeneous

equation ...

Martin Scharlemann, Transverse Whitehead triangulations ...

Gary Joseph Sherman, A lower bound for the number of conjugacy classes in a

finite nilpotent group

Richard Arthur Shoop, The Lebesgue constants for $\left(f, d_{n}\right)$-summability .

Stuart Jay Sidney, Functions which operate on the real part of a uniform

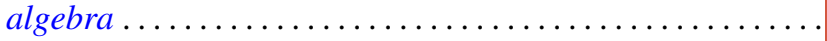

Tim Eden Traynor, The group-valued Lebesgue decomposition 